\title{
Which countries are the best in tobacco control? A quantitative analysis of the MPOWER 2017
}

Gholamreza Heydari

Keywords: mpower, tobacco control, global health

https://doi.org/10.29392/joghr.3.e2019039

\section{Journal of Global Health Reports}

Vol. 3, 2019

\begin{abstract}
Background
The World Health Organization (WHO) introduced a package of advice to countries including six main policies (MPOWER) to control tobacco use. Our objective was to perform a quantitative analysis of MPOWER in countries and regions to create competition between parties.
\end{abstract}

\section{Methods}

This cross-sectional study was based on the MPOWER data 2017, with countries assessed using a validated check list of 10 criteria (possible maximum score 37). The scores were summed and presented in a descending order for 6 regions.

\section{Results}

The 23 countries which had at least $85 \%$ of total score (i.e., at least 32 from 37) were: United Kingdom, Turkey and Costa Rica (36); Brazil, Panama and Australia (35), Surinam, Colombia, Iran and New Zealand (34); Portugal, Russia, Ireland, Seychelles, Mauritius, Canada, Uruguay and Argentina (33); and Romania, Estonia, Denmark, Spain and Norway (32).

\section{Conclusions}

These 23 countries may present as the best model. Comparison of scores of different countries in this respect can be beneficial since it creates a challenge for the countries to achieve a higher rank.

The need for implementation of tobacco control programs is undeniable due to hazards from smoking. ${ }^{1}$ Tobacco use remains the first preventable cause of morbidity and mortality from chronic diseases worldwide, and the rate of morbidity and mortality is rising due to smoking-related diseases. $^{2,3}$ Therefore, the first and the most important strategy to confront this is the comprehensive implementation of tobacco control programs. ${ }^{4,5}$ In this regard, the WHO negotiated the Framework Convention on Tobacco Control (FCTC) treaty in 2003 and so far, 181 countries have ratified it. ${ }^{6}$ In 2008 , a package was proposed to be implemented and included six main components, namely: monitor tobacco use and prevention policies, protect people from tobacco smoke, offer help to quit tobacco use, warn people about the dangers of tobacco, enforce bans on tobacco advertising, promotion and sponsorship, and raise taxes on tobacco. ${ }^{7}$ Global experiences have revealed that implementation of the above-mentioned six strategies can effectively decrease the rate of consumption and resultantly the consequences and complications of tobacco use. ${ }^{8-1011}$ Some studies showed that this type of analysis may create a challenge between countries to improve their status on tobacco control. ${ }^{12,13}$ A study in 2015 showed that fifteen countries the highest scores on tobacco control worldwide. ${ }^{14}$
This study sought to perform a quantitative analysis of the above-mentioned report and track the status of tobacco control programs of different countries and the six WHO regions in this regard to create competition between parties with introducing best parties and may motivate countries as a stimulant to be more active on tobacco control.

\section{METHODS}

This cross-sectional study was conducted in the second half of 2017 using a researcher-made checklist that was validated in previous two studies. ${ }^{13,14}$ The data for each country related to 10 variables were completed and recorded including 7 MPOWER recommendations, 2 compliances, and the prevalence of tobacco use based on the WHO 2017 report. For the 5 -item variables, the score was from 0 to 4 ; and for the 4 -item variables, the score was $0-3$. Thus, the range of total scores was calculated from zero to 37 . The checklist was crossed-check and finalized by the panel of experts at a meeting in Tobacco Prevention and Control Research Center and the scores were confirmed and approved. The scores of each country were finalized and arranged according to the six zones of the WHO in separate tables. 
The checklist and the scoring system used are presented in Table 1.

\section{RESULTS}

Countries which had at least $85 \%$ of total score (32 out of 37) were Seychelles and Mauritius at 33 each (i.e. two from 47 countries, representing $4.2 \%$ of the WHO Regional Office for Africa (AFRO) region), Costa Rica 36, Brazil and Panama 35, Surinam and Colombia 34, Canada, Uruguay and Argentina 33 (ie. 8 from 35 countries, representing $22.8 \%$ of the WHO Regional Office for the Americas AMRO region), United Kingdom and Turkey 36, Portugal, Russia, Ireland 33, Romania, Estonia Denmark, Spain and Norway 32 (ie. 10 from 53 countries, representing $18.8 \%$ of the WHO Regional Office for Europe (EURO) region), Iran 34 (i.e. 1 from 22 countries, representing $4.5 \%$ of the WHO Regional Office of the Eastern Mediterrean (EMRO) region), and Australia 35, New Zealand 34 (2 from 27 countries, representing 7.4\% of the WHO Western Pacific Regional Office (WPRO) region). There was no country in the WHO South East Asia Regional Office (SEARO) region. The 23 countries and their scores (based on the 10 indicators) with differences from 2015 are show in Table 2.

The highest mean points were scored by Europe (26.41). Other regions are as follows: South-East Asia (25.09), West Pacific (24.88), America (22.05), East Mediterranean region (21.40) and Africa (17.40). The mean difference was significant $(P<0.05)$. A negative correlation between low smoking prevalence and high total score was observed $(P=0.02)$.

Countries which had less than $30 \%$ of total score (11 out of37) were: Sao Tome, Malawi, and Lesotho 10, Guinea Bissau, Equatorial Guinea, and Angola 8, South Sudan, Burundi, and Mauritania 7, Central Africa 6 (ie. 10 from 47 countries, representing $21 \%$ of AFRO region); Saint Vincent 9, Saint Kitts 8, Dominica 7 (ie. 3 from 35 countries, representing $8 \%$ of AMRO region); Monaco 7, (ie. 1 from 53 countries, representing $2 \%$ of EURO region); and Somali 7 (ie. 1 from 22 countries, representing 4\% of EMRO region). There were no countries from SEARO and WPRO regions.

This finding mentioned that Africa region which had fewer countries in top scores (4\%) had highest countries in low scores (21\%) and it showed vice versa for European region (19\%-2\%).

\section{DISCUSSION}

This study showed that none of the countries had full scores in the tobacco control programs; however, 23 countries (Seychelles, Mauritius, Costa Rica, Brazil, Panama, Surinam, Colombia, Canada, Uruguay, Argentina, United Kingdom, Turkey, Portugal, Russia, Ireland, Romania, Estonia, Denmark, Spain, Norway, Iran, Australia and New Zealand) had a superior status according to the 2017 MPOWER report. These 23 countries may present a best model for other parties in the implementation and enforcement of tobacco control programs. Comparison of scores of different countries in this respect can be beneficial since it creates a challenge for the health policy makers to find weakness of the tobacco control programs to work on it. Also, Europe had a superior position over others as well.

In 2015, fifteen countries, which acquired the highest scores (85\% of total 37) included Panama and Turkey with 35, Brazil and Uruguay with 34, Ireland, United Kingdom, Iran, Brunei, Argentina and Costa Rica with 33 and Australia, Nepal, Thailand, Canada and Mauritius with 32 points. ${ }^{14}$ The comparison between these two studies shows that 4 countries (Brunei, Nepal, Thailand and Mauritius) left and 12 new countries are added to this group. It may a create competition between countries to have more focus on tobacco control.

In pages 136 to 149 of the 2017 MPOWER report ${ }^{11}$ we found 9 countries with lowest smoking prevalence in the world, $5 \%$ or less including Ethiopia, Ghana and Panama (3\%), Ecuador (4\%), Benin, Barbados, Eritrea, Niger and Nigeria (5\%) and based on Table 3 to Table 8 the total score of those are $22,22,35,31,26,23,18,23$ and 19, respectively. It shows that among these, only Panama is in top 23. It might be due to other reasons which can be explored in future studies, but we found a significant negative correlation between low smoking prevalence and high total score.

Since the scores were very much close and most countries had a one-point difference, more precise implementation of each strategy and publishing a more thorough report may change the scores and consequently the ranking of countries in this respect. [Tables 3 to Table-8](402727e0-132c-462c-8a6dc00a88f2291d)[](eacffb78-9075-4fc2-8928-99349f9ba4c0) show that all of the regions had higher total scores compared with 2015 including Africa +52 , America +59 , SEAR +35 , Europe +109 , EMR +43 and WEPR +43 . Highest mean score of this is belonging to SEAR with 3.18 and then 2.05 for Europe. It is notable that SEAR has no country in the top 23 but it has best improvement regionally. Also Table 3 to Table 8 show that highest score improvement belong to Timor Leste +13 , Cambodia +12 , Elsalvador and Romania +9, Uganda, Ruanda, Syrian Arab Republic +8 and highest reduction belong to Cameron -7 , Luxemburg -6 , San Marino, Libya, Swaziland -5 .

The superior position of European countries in this regard has also been mentioned in a study by Joossens. ${ }^{15}$ Except in the Eastern Mediterranean region, no such a study has been conducted in other parts of the world ${ }^{16}$; therefore, this can be an interesting research topic for future studies and the results can be used to create a challenge and a competition among countries in an effort to gain a better ranking.

\section{LIMITATIONS}

Firstly, the study was limited by not randomizing selection. In addition, we did not ensure the report on MPOWER which was sent from country to WHO was aligned with the real status of tobacco control in the society.

\section{CONCLUSION}

Countries perform differently with regard to tobacco control programs and these 23 countries possibly present the best model. Comparison of scores of different countries in 
Table 1. MPOWER WHO score on tobacco control check list based on WHO report 2017

\begin{tabular}{|c|c|}
\hline & Score \\
\hline Adult daily smoking prevalence & 4 \\
\hline Estimates not available & 0 \\
\hline $30 \%$ or more & 1 \\
\hline From $20 \%$ to $29 \%$ & 2 \\
\hline From $15 \%$ to $19 \%$ & 3 \\
\hline Less than $15 \%$ & 4 \\
\hline Monitoring: prevalence data & 3 \\
\hline No known data or no recent data or data that is not both recent and representative & 0 \\
\hline Recent and representative data for either adults or youth & 1 \\
\hline Recent and representative data for both adults and youth & 2 \\
\hline Recent, representative and periodic data for both adults and youth & 3 \\
\hline Smoke-free policies & 4 \\
\hline Data not reported & 0 \\
\hline Up to two public places completely smoke-free & 1 \\
\hline Three to five public places completely smoke-free & 2 \\
\hline Six and seven public places completely smoke-free & 3 \\
\hline All public places completely smoke-free & 4 \\
\hline Cessation program & 4 \\
\hline Data not reported & 0 \\
\hline None & 1 \\
\hline NRT and/or some cessation services (neither cost-covered) & 2 \\
\hline NRT and/or some cessation services (at least one of which is cost-covered) & 3 \\
\hline National quit line, and both NRT and some cessation services cost-covered & 4 \\
\hline Health warning on cigarette packages & 4 \\
\hline Data no reported & 0 \\
\hline No warnings or small warnings & 1 \\
\hline Medium size warnings missing some appropriate characteristics & 2 \\
\hline Medium size warnings with all appropriate characteristics & 3 \\
\hline Large warnings with all appropriate characteristics & 4 \\
\hline Anti-tobacco mass media campaigns & 4 \\
\hline Data not reported & 0 \\
\hline No campaign conducted & 1 \\
\hline Campaign conducted with 1-4 appropriate characteristics & 2 \\
\hline Campaign conducted with 5-6 appropriate characteristics & 3 \\
\hline Campaign conducted with all appropriate characteristics & 4 \\
\hline Advertising bans & 4 \\
\hline Data not reported & 0 \\
\hline Complete absence of ban print media & 1 \\
\hline Ban on national television, radio and print media only & 2 \\
\hline Ban on national television, radio and print media & 3 \\
\hline Ban on all forms of direct and indirect advertising & 4 \\
\hline Taxation & 4 \\
\hline Data not reported & 0 \\
\hline$\leq 25 \%$ of retail price is tax & 1 \\
\hline $26-50 \%$ of retail price is tax & 2 \\
\hline $51-75 \%$ of retail price is tax & 3 \\
\hline$>75 \%$ of retail price is tax & 4 \\
\hline Compliance bans on advertising & 3 \\
\hline
\end{tabular}




\begin{tabular}{lc}
\hline & Score \\
\hline Complete compliance (8/10 to $10 / 10)$ & 3 \\
Moderate compliance (3/10 to $7 / 10)$ & 2 \\
Minimal compliance (0/10 to $2 / 10)$ & 1 \\
Not report & 0 \\
Compliance smoke-free policy & 3 \\
Complete compliance (8/10 to $10 / 10)$ & 3 \\
Moderate compliance (3/10 to $7 / 10)$ & 2 \\
Minimal compliance (0/10 to $2 / 10)$ & 1 \\
Not report & \\
Total & \\
\hline
\end{tabular}

this respect can be beneficial since it creates a challenge for the countries to achieve a higher rank. Based on WHO documents we have a simple validated view of ranking of countries and regions regarding tobacco control which may create a challenge to improve this status. Comparison of scores of different countries in this respect can be motivate the health policy makers to find weakness of the tobacco control programs to work on it.

\section{CORRESPONDENCE TO:}

Gholamreza Heydari

Tobacco Prevention and Control Research Center

Niavaran, Darabad, PC19556

Tehran

Iran

heydarigh@sbmu.ac.ir 
Table 2. MPOWER WHO score on tobacco control for top 23 countries

\begin{tabular}{|c|c|c|c|c|c|c|c|c|c|c|c|c|}
\hline Country & $\begin{array}{l}\text { Smoking } \\
\text { prevalence }\end{array}$ & Monitoring & $\begin{array}{l}\text { Smoke- } \\
\text { free } \\
\text { policies }\end{array}$ & $\begin{array}{l}\text { Smoke-free policy } \\
\text { compliance }\end{array}$ & $\begin{array}{l}\text { Cessation } \\
\text { programs }\end{array}$ & $\begin{array}{l}\text { Health warning on } \\
\text { cigarette packages }\end{array}$ & $\begin{array}{l}\text { Mass media } \\
\text { campaigns }\end{array}$ & $\begin{array}{l}\text { Advertising } \\
\text { bans }\end{array}$ & $\begin{array}{l}\text { Advertising bans } \\
\text { compliance }\end{array}$ & Taxation & Score & $\begin{array}{l}\text { Difference } \\
\text { from } 2015\end{array}$ \\
\hline $\begin{array}{l}\text { Costa } \\
\text { Rica }\end{array}$ & 4 & 4 & 4 & 3 & 3 & 4 & 4 & 3 & 3 & 3 & 36 & +3 \\
\hline $\begin{array}{l}\text { United } \\
\text { Kingdom }\end{array}$ & 3 & 4 & 4 & 3 & 4 & 4 & 4 & 3 & 3 & 4 & 36 & +3 \\
\hline Turkey & 2 & 4 & 4 & 3 & 4 & 4 & 4 & 4 & 3 & 4 & 36 & +1 \\
\hline $\begin{array}{l}\text { New } \\
\text { Zealand }\end{array}$ & 3 & 4 & 4 & 3 & 4 & 4 & 4 & 3 & 3 & 3 & 35 & +7 \\
\hline Brazil & 4 & 4 & 4 & 3 & 4 & 4 & 2 & 4 & 3 & 3 & 35 & +1 \\
\hline Panama & 4 & 4 & 4 & 3 & 4 & 4 & 2 & 4 & 3 & 3 & 35 & - \\
\hline Suriname & 3 & 3 & 4 & 3 & 3 & 4 & 4 & 4 & 3 & 3 & 34 & +4 \\
\hline Colombia & 4 & 4 & 4 & 3 & 3 & 3 & 4 & 4 & 3 & 2 & 34 & +3 \\
\hline Australia & 4 & 4 & 4 & 0 & 4 & 4 & 4 & 3 & 4 & 3 & 34 & +2 \\
\hline Iran (IR) & 4 & 4 & 4 & 3 & 4 & 4 & 3 & 4 & 3 & 1 & 34 & +1 \\
\hline Seychelles & 3 & 3 & 4 & 3 & 3 & 4 & 4 & 3 & 3 & 3 & 33 & +5 \\
\hline Portugal & 3 & 4 & 3 & 3 & 3 & 4 & 4 & 3 & 3 & 3 & 33 & +5 \\
\hline Russian F. & 2 & 4 & 4 & 3 & 3 & 4 & 3 & 4 & 3 & 3 & 33 & +3 \\
\hline Canada & 4 & 4 & 4 & 3 & 4 & 4 & 1 & 3 & 3 & 3 & 33 & +1 \\
\hline Mauritius & 3 & 3 & 3 & 3 & 3 & 4 & 4 & 4 & 3 & 3 & 33 & +1 \\
\hline Uruguay & 4 & 4 & 4 & 3 & 3 & 4 & 1 & 4 & 3 & 3 & 33 & -1 \\
\hline Argentina & 3 & 4 & 4 & 3 & 3 & 4 & 3 & 3 & 3 & 4 & 33 & - \\
\hline Ireland & 3 & 4 & 4 & 0 & 4 & 4 & 4 & 3 & 3 & 4 & 33 & - \\
\hline Romania & 2 & 4 & 4 & 3 & 3 & 4 & 3 & 3 & 3 & 3 & 32 & +9 \\
\hline Estonia & 2 & 4 & 1 & 3 & 4 & 4 & 4 & 3 & 3 & 4 & 32 & +7 \\
\hline Denmark & 3 & 4 & 1 & 3 & 4 & 4 & 4 & 3 & 3 & 3 & 32 & +5 \\
\hline Spain & 3 & 4 & 4 & 3 & 3 & 3 & 1 & 4 & 3 & 4 & 32 & +2 \\
\hline Norway & 3 & 4 & 4 & 3 & 3 & 3 & 4 & 3 & 3 & 3 & 32 & +1 \\
\hline
\end{tabular}


Table 3. Africa Region countries ranked by total MPOWER WHO score on tobacco control in 2017*

\begin{tabular}{|c|c|c|c|c|c|c|c|c|c|c|c|c|}
\hline Country & $\begin{array}{l}\text { Smoking } \\
\text { prevalence }\end{array}$ & Monitoring & $\begin{array}{l}\text { Smoke- } \\
\text { free } \\
\text { policies }\end{array}$ & $\begin{array}{c}\text { Smoke-free } \\
\text { policy } \\
\text { compliance }\end{array}$ & $\begin{array}{l}\text { Cessation } \\
\text { programs }\end{array}$ & $\begin{array}{l}\text { Health warning on } \\
\text { cigarette packages }\end{array}$ & $\begin{array}{l}\text { Mass media } \\
\text { campaigns }\end{array}$ & $\begin{array}{l}\text { Advertising } \\
\text { bans }\end{array}$ & $\begin{array}{l}\text { Advertising bans } \\
\text { compliance }\end{array}$ & Taxation & Score & $\begin{array}{l}\text { Difference } \\
\text { from } 2015\end{array}$ \\
\hline Seychelles & 3 & 3 & 4 & 3 & 3 & 4 & 4 & 3 & 3 & 3 & 33 & +5 \\
\hline Mauritias & 3 & 3 & 3 & 3 & 3 & 4 & 4 & 4 & 3 & 3 & 33 & +1 \\
\hline Senegal & 4 & 3 & 3 & 0 & 4 & 4 & 1 & 4 & 3 & 2 & 28 & +6 \\
\hline Uganda & 4 & 4 & 4 & 0 & 3 & 3 & 1 & 4 & 3 & 1 & 27 & +8 \\
\hline Burkin faso & 4 & 2 & 4 & 2 & 2 & 4 & 1 & 3 & 3 & 2 & 27 & +4 \\
\hline Benin & 4 & 2 & 3 & 1 & 3 & 2 & 1 & 3 & 2 & 1 & 26 & +1 \\
\hline Kenya & 4 & 3 & 1 & 0 & 3 & 3 & 4 & 4 & 0 & 3 & 25 & +2 \\
\hline Madagascar & 0 & 1 & 4 & 2 & 2 & 4 & 1 & 4 & 3 & 4 & 25 & +1 \\
\hline Togo & 4 & 3 & 2 & 2 & 1 & 3 & 1 & 4 & 3 & 1 & 24 & +3 \\
\hline Niger & 4 & 1 & 2 & 0 & 2 & 4 & 1 & 4 & 3 & 2 & 23 & - \\
\hline South Africa & 3 & 4 & 1 & 0 & 3 & 1 & 1 & 3 & 3 & 3 & 22 & +5 \\
\hline Ethiopia & 4 & 2 & 0 & 2 & 3 & 1 & 3 & 3 & 3 & 1 & 22 & +4 \\
\hline Ghana & 4 & 2 & 0 & 0 & 3 & 3 & 1 & 4 & 3 & 2 & 22 & -3 \\
\hline Namibia & 3 & 1 & 4 & 0 & 3 & 4 & 1 & 3 & 0 & 2 & 21 & -3 \\
\hline Cameron & 0 & 3 & 2 & 0 & 3 & 3 & 4 & 3 & 0 & 1 & 21 & -7 \\
\hline Mozambique & 4 & 2 & 1 & 2 & 2 & 1 & 1 & 3 & 2 & 2 & 20 & +2 \\
\hline Nigeria & 4 & 2 & 1 & 0 & 3 & 3 & 1 & 4 & 0 & 1 & 19 & +6 \\
\hline Gambia & 4 & 1 & 1 & 0 & 1 & 2 & 1 & 3 & 3 & 3 & 19 & +6 \\
\hline Congo & 3 & 1 & 4 & 0 & 3 & 2 & 1 & 3 & 0 & 2 & 19 & -1 \\
\hline Comoros & 4 & 3 & 2 & 1 & 1 & 2 & 1 & 1 & 2 & 2 & 19 & - \\
\hline Rwanda & 4 & 2 & 1 & 0 & 2 & 2 & 0 & 1 & 3 & 3 & 18 & +8 \\
\hline Caboverde & 4 & 4 & 1 & 0 & 3 & 1 & 1 & 3 & 0 & 1 & 18 & +6 \\
\hline Botswan & 3 & 2 & 1 & 0 & 3 & 2 & 1 & 3 & 1 & 1 & 18 & +5 \\
\hline Zimbabwe & 4 & 2 & 2 & 2 & 3 & 1 & 1 & 1 & 0 & 2 & 18 & +4 \\
\hline Eritrea & 4 & 1 & 1 & 0 & 1 & 2 & 0 & 4 & 3 & 3 & 18 & +3 \\
\hline Mali & 4 & 2 & 1 & 0 & 3 & 2 & 1 & 3 & 0 & 2 & 18 & - \\
\hline Zambia & 4 & 2 & 3 & 1 & 2 & 1 & 1 & 1 & 0 & 2 & 17 & +2 \\
\hline UR Tanzania & 4 & 2 & 1 & 0 & 3 & 1 & 0 & 3 & 1 & 2 & 17 & +1 \\
\hline Chad & 0 & 1 & 4 & 0 & 2 & 4 & 0 & 4 & 0 & 2 & 17 & -3 \\
\hline Swaziland & 4 & 2 & 1 & 0 & 3 & 2 & 0 & 3 & 0 & 2 & 17 & -5 \\
\hline Algeria & 4 & 2 & 2 & 0 & 2 & 1 & 0 & 3 & 0 & 2 & 16 & -4 \\
\hline
\end{tabular}




\begin{tabular}{|c|c|c|c|c|c|c|c|c|c|c|c|c|}
\hline Country & $\begin{array}{c}\text { Smoking } \\
\text { prevalence }\end{array}$ & Monitoring & $\begin{array}{c}\begin{array}{c}\text { Smoke- } \\
\text { free } \\
\text { policies }\end{array}\end{array}$ & $\begin{array}{c}\text { Smoke-free } \\
\text { policy } \\
\text { compliance }\end{array}$ & $\begin{array}{l}\text { Cessation } \\
\text { programs }\end{array}$ & $\begin{array}{l}\text { Health warning on } \\
\text { cigarette packages }\end{array}$ & $\begin{array}{l}\text { Mass media } \\
\text { campaigns }\end{array}$ & $\begin{array}{c}\text { Advertising } \\
\text { bans }\end{array}$ & $\begin{array}{l}\text { Advertising bans } \\
\text { compliance }\end{array}$ & Taxation & Score & $\begin{array}{l}\text { Difference } \\
\text { from } 2015\end{array}$ \\
\hline Gabon & 0 & 2 & 2 & 0 & 2 & 3 & 1 & 3 & 0 & 1 & 14 & -2 \\
\hline Guinea & 0 & 1 & 2 & 0 & 2 & 2 & 1 & 4 & 0 & 1 & 13 & - \\
\hline Sierra Leone & 3 & 1 & 1 & 0 & 1 & 1 & 3 & 1 & 0 & 1 & 12 & +2 \\
\hline Cote D'ivore & 0 & 1 & 1 & 0 & 3 & 1 & 3 & 1 & 0 & 2 & 12 & - \\
\hline Liberia & 4 & 2 & 1 & 0 & 2 & 1 & 0 & 1 & 0 & 1 & 12 & - \\
\hline D.R. Congo & 0 & 1 & 2 & 0 & 2 & 2 & 1 & 2 & 0 & 2 & 12 & -2 \\
\hline Sao Tome & 0 & 1 & 1 & 0 & 3 & 1 & 0 & 3 & 0 & 1 & 10 & +1 \\
\hline Malawi & 4 & 1 & 1 & 0 & 1 & 1 & 0 & 1 & 0 & 1 & 10 & -2 \\
\hline Guinea B. & 0 & 1 & 1 & 0 & 2 & 1 & 1 & 3 & 0 & 1 & 8 & +2 \\
\hline E. Guinea & 0 & 1 & 1 & 0 & 1 & 1 & 0 & 1 & 0 & 3 & 8 & +1 \\
\hline Angola & 0 & 1 & 2 & 0 & 2 & 1 & 1 & 1 & 0 & 0 & 8 & +1 \\
\hline South Sudan & 0 & 1 & 1 & 0 & 0 & 1 & 1 & 1 & 0 & 2 & 7 & +4 \\
\hline Burundi & 0 & 1 & 1 & 0 & 1 & 1 & 0 & 1 & 0 & 2 & 7 & - \\
\hline Mauritania & 0 & 1 & 1 & 0 & 1 & 1 & 0 & 1 & 0 & 2 & 7 & -3 \\
\hline $\begin{array}{l}\text { Central } \\
\text { African } \\
\text { Republic }\end{array}$ & 0 & 1 & 1 & 0 & 2 & 1 & 0 & 1 & 0 & 0 & 6 & -4 \\
\hline Total & & & & & & & & & & & 818 & +52 \\
\hline
\end{tabular}

"The mean score of the region=17.40 
Table 8. Western Pacific Region countries ranked by total MPOWER WHO score on tobacco control in 2017*

\begin{tabular}{|c|c|c|c|c|c|c|c|c|c|c|c|c|}
\hline Country & $\begin{array}{c}\text { Smoking } \\
\text { prevalence }\end{array}$ & Monitoring & $\begin{array}{l}\text { Smoke- } \\
\text { free } \\
\text { policies }\end{array}$ & $\begin{array}{l}\text { Smoke-free policy } \\
\text { compliance }\end{array}$ & $\begin{array}{l}\text { Cessation } \\
\text { programs }\end{array}$ & $\begin{array}{l}\text { Health warning on } \\
\text { cigarette packages }\end{array}$ & $\begin{array}{l}\text { Mass media } \\
\text { campaigns }\end{array}$ & $\begin{array}{c}\text { Advertising } \\
\text { bans }\end{array}$ & $\begin{array}{l}\text { Advertising bans } \\
\text { compliance }\end{array}$ & Taxation & Score & $\begin{array}{l}\text { Difference } \\
\text { from } 2015\end{array}$ \\
\hline $\begin{array}{l}\text { New } \\
\text { Zealand }\end{array}$ & 3 & 4 & 4 & 3 & 4 & 4 & 4 & 3 & 3 & 3 & 35 & +7 \\
\hline Australia & 4 & 4 & 4 & 0 & 4 & 4 & 4 & 3 & 4 & 3 & 34 & +2 \\
\hline Brunei & 4 & 4 & 4 & 0 & 4 & 4 & 4 & 3 & 4 & 0 & 31 & -2 \\
\hline Cambodia & 3 & 4 & 4 & 2 & 2 & 4 & 4 & 3 & 3 & 1 & 30 & +12 \\
\hline Philippines & 3 & 4 & 1 & 1 & 3 & 4 & 4 & 3 & 3 & 3 & 29 & +3 \\
\hline Vietnam & 3 & 4 & 3 & 2 & 1 & 4 & 4 & 3 & 3 & 2 & 29 & +1 \\
\hline Korea R. & 2 & 4 & 1 & 3 & 4 & 3 & 4 & 1 & 3 & 3 & 28 & +5 \\
\hline Tonga & 2 & 2 & 3 & 3 & 3 & 2 & 4 & 3 & 3 & 3 & 28 & +5 \\
\hline Malaysia & 3 & 4 & 1 & 0 & 3 & 4 & 4 & 3 & 3 & 3 & 28 & +1 \\
\hline Singapore & 4 & 4 & 2 & 0 & 4 & 4 & 4 & 3 & 0 & 3 & 28 & +1 \\
\hline Fiji & 3 & 3 & 2 & 3 & 3 & 4 & 4 & 3 & 3 & 2 & 28 & 0 \\
\hline Mongolia & 2 & 4 & 4 & 2 & 3 & 4 & 1 & 4 & 3 & 1 & 28 & -3 \\
\hline Samoa & 2 & 3 & 3 & 3 & 3 & 4 & 5 & 3 & 3 & 3 & 27 & -1 \\
\hline Palau & 4 & 4 & 3 & 3 & 3 & 1 & 0 & 3 & 3 & 3 & 27 & +2 \\
\hline Vanuatu & 4 & 3 & 1 & 0 & 3 & 4 & 1 & 4 & 3 & 3 & 26 & +7 \\
\hline Lao R. & 2 & 4 & 4 & 3 & 1 & 4 & 0 & 3 & 3 & 1 & 25 & +4 \\
\hline China & 2 & 3 & 1 & 1 & 3 & 2 & 4 & 3 & 2 & 3 & 24 & 3 \\
\hline Nauru & 1 & 2 & 4 & 2 & 2 & 2 & 1 & 3 & 3 & 3 & 23 & +4 \\
\hline Kiribati & 1 & 3 & 2 & 0 & 3 & 3 & 4 & 4 & 0 & 2 & 22 & -2 \\
\hline $\begin{array}{l}\text { Cook } \\
\text { Island }\end{array}$ & 3 & 4 & 2 & 0 & 3 & 3 & 1 & 3 & 0 & 3 & 22 & +1 \\
\hline Tuvalu & 0 & 1 & 1 & 2 & 3 & 2 & 1 & 4 & 3 & 3 & 20 & +1 \\
\hline Solomon I. & 0 & 1 & 1 & 2 & 3 & 4 & 0 & 3 & 3 & 2 & 19 & -4 \\
\hline Marshal I. & 0 & 1 & 4 & 2 & 3 & 1 & 1 & 1 & 3 & 3 & 19 & 0 \\
\hline Japan & 3 & 4 & 1 & 0 & 3 & 2 & 1 & 1 & 0 & 3 & 18 & +1 \\
\hline Niue & 4 & 2 & 1 & 0 & 3 & 1 & 0 & 1 & 0 & 4 & 16 & 0 \\
\hline Micronesia & 0 & 3 & 1 & 2 & 3 & 1 & 1 & 1 & 0 & 3 & 15 & +1 \\
\hline Papa N.G. & 1 & 1 & 4 & 0 & 1 & 1 & 1 & 3 & 1 & 2 & 13 & -3 \\
\hline Total & & & & & & & & & & & 672 & +43 \\
\hline
\end{tabular}

"The mean score of the region=24.88. 


\section{REFERENCES}

1. Ng M, Freeman MK, Fleming TD, et al. Smoking prevalence and cigarette consumption in 187 countries, 1980-2012. JAMA. 2014;311(2):183-192. do i:10.1001/jama.2013.284692

2. Mathers CD, Loncar D. Projections of global mortality and burden of disease from 2002 to 2030 . PLoS Med. 2006;3(11):e442. doi:10.1371/journal.pme d.0030442

3. Peto R, Lopez AD, Boreham J, Thun M, Heath C, Doll R. Mortality from smoking worldwide. $\mathrm{Br} \mathrm{Med}$ Bull. 1996;52(1):12-21. doi:10.1093/oxfordjournals.b $\underline{\text { mb.a011519 }}$

4. Levine R, Kinder M. Millions Saved: Proven Success in Global Health. Routledge; 2006.

5. Battling big tobacco: Mike Wallace talks to the highest-ranking tobacco whistleblower. CBS News. Published 2005. Accessed December 5, 2007. http://w ww.cbsnews.com/2100-500164-162-666867.html

6. World Health Organization. WHO Framework Convention on Tobacco Control. Accessed February 27, 2005. http://www.who.int/fctc/text_download/en/

7. World Health Organization. Tobacco Free Initiative. Accessed December 2013. http://www.who.int/entity/ tobacco/mpower/en/

8. Guindon GE, Boisclair D. Past, Current and Future Trends in Tobacco Use. World Bank; 2003. Accessed December 5, 2007. https://openknowledge.worldban k.org/handle/10986/13726

9. Basu S, Glantz S, Bitton A, Millett C. The effect of tobacco control measures during a period of rising cardiovascular disease risk in India: a mathematical model of myocardial information and stroke. PLoS Med. 2013;10(7):e1001480. doi:10.1371/journal.pme d.1001480
10. Levy DT, Ellis JA, Mays D, Huang AT. Smokingrelated deaths averted due to three years of policy progress. Bull World Health Organ.

2013;91(7):509-518. doi:10.2471/blt.12.113878

11. World Health Organization. WHO Report on the Global Tobacco Epidemic 2017. WHO; 2017. Accessed July 19, 2017. http://www.who.int/tobacco/global_rep ort/2017/en/

12. Heydari G, Talischi F, Algouhmani H, Lando HA, Ahmady AE. WHO MPOWER tobacco control scores in the Eastern Mediterranean countries based on the 2011 report. EMHJ. 2013;19:314-319. doi:10.26719/20 $\underline{13.19 .4 .314}$

13. Heydari G, Ebn Ahmady A, Lando HA, Shadmehr MB, Fadaizadeh L. The second study on WHO MPOWER tobacco control scores in the Eastern Mediterranean Countries based on the 2013 report: improvements during two years. Arch Iran Med. 2014;17:621-625.

14. Heydari G, Chamyani F, Masjedi M, Fadaizadeh L. Comparison of tobacco control programs worldwide: a quantitative analysis of the 2015 World Health Organization MPOWER report. Int J Prev Med. 2016;7(1):127. doi:10.4103/2008-7802.195562

15. Joossens L, Raw M. The tobacco control scale: a new scale to measure country activity. Tob Control. 2006;15(3):247-253. doi:10.1136/tc.2005.015347

16. Heydari G, Talischi F, Masjedi MR, Alguomani H, Joossens L, Ghafari M. Comparison of tobacco control Policies in the Eastern Mediterranean countries based on tobacco control scale score. East Mediterr Health J. 2012;18:804-810. doi:10.26719/2012.18.8.803 